\section{Cureus}

Received 04/29/2019

Review began 05/02/2019

Review ended 05/02/2019

Published 05/07/2019

\section{(c) Copyright 2019}

Shahid et al. This is an open access article distributed under the terms of the Creative Commons Attribution License CC-BY 3.0., which permits unrestricted use, distribution, and reproduction in any medium, provided the original author and source are credited.

\title{
The First Case of Rowell Syndrome with Lupus Nephritis and Lobar Pneumonia in a Male Child Reported in Pakistan
}

\author{
Sumreen Shahid ${ }^{1}$, Maria Khan ${ }^{2}$, Laila Tul Qadar ${ }^{2}$, Manahil Akmal ${ }^{2}$, Ammarah Jamal ${ }^{3}$ \\ 1. Miscellaneous, Civil Hospital Karachi, Karachi, PAK 2. Internal Medicine, Dow University of Health \\ Sciences, Karachi, PAK 3. Pediatrics, Civil Hospital Karachi, Dow University of Health Sciences, Karachi, \\ PAK
}

Corresponding author: Maria Khan, mariajawaid96@gmail.com

\section{Abstract}

Rowell syndrome (RS) is a rare entity that presents with lupus erythematosus (LE), erythema multiforme (EM) like lesions and characteristic immunological findings including positive rheumatoid factor (RF), speckled antinuclear antibody (ANA) and positive anti-Ro or anti-La antibodies in the serum. Only a few cases have been reported in the literature, mostly in middle-aged women. Our case is about an 11-year-old male child who had a history of pustular rashes over the whole body with scattered targetoid lesions for the past few months and later developed respiratory distress and swelling of the body. He was extensively investigated and confirmed on specific positive immunochemistry markers as RS along with lobar pneumonia (LP) and lupus nephritis. The co-existence of lobar pneumonia in our patient was a unique feature that has not been previously reported.

Categories: Dermatology, Pediatrics, Rheumatology

Keywords: rowell syndrome, lupus erythematosus, systemic lupus erythematosus, lupus nephritis, lobar pneumonia, erythema multiforme

\section{Introduction}

Rowell syndrome (RS), first described in 1922 by Scholtz et al., was later characterized as a separate syndrome by Rowell and his colleagues in 1963 when they encountered four women who were suffering from systemic lupus erythematosus (SLE) and had developed rashes suggestive of erythema multiforme (EM) [1-2].

Since then 33 cases of RS have been reported in the literature, mostly affecting middle-aged women [3]. In 2008, Zeitouni et al. defined the major diagnostic criteria of RS consisting of preexisting lupus erythematosus (LE) getting superimposed by EM and speckled pattern of antinuclear antibody (ANA), along with at least one minor criterion consisting of positive rheumatoid factor (RF), chilblains or the presence of anti-Ro or anti-La antibodies [4].

We report a rare case of RS at an unusual age of 11 years with an unfamiliar presentation of lobar pneumonia (LP).

\section{Case Presentation}

An 11-year-old, previously well, vaccinated male child was admitted in the pediatric ward of Dr. Ruth KM Pfau, Civil Hospital Karachi (CHK) with a two-day history of fever, cough, and abdominal distension followed by respiratory distress. His illness started seven months earlier

How to cite this article

Shahid S, Khan M, Qadar L, et al. (May 07, 2019) The First Case of Rowell Syndrome with Lupus Nephritis and Lobar Pneumonia in a Male Child Reported in Pakistan. Cureus 11(5): e4604. DOI 10.7759 /cureus. 4604 


\section{Cureus}

with the eruption of pruritic, blistering rashes all over the body accompanied by a pussy discharge. At the time of onset, the rash was associated with low-grade fever, skin hyperpigmentation, oral ulcers and joint pains without any swelling or movement restriction. The rash usually appeared in successive generations on the sun-exposed regions progressing into a darkly pigmented scar over a period of one week. He consulted various doctors and was even hospitalized once for the rash but showed only temporary improvement to intravenous antibiotics and prednisolone. On examination, he showed obvious signs of respiratory distress with a respiratory rate of 40 breaths/min and a heart rate of 90 beats/min. Examination of the chest revealed signs consistent with left-sided LP. In addition, he had a scaly rash with occasional targetoid lesions all over the body associated with peeling of the skin (Figure 1), ruptured blisters, loss of fingernails (Figure 2) and sparse, brittle and depigmented hairs. He had hepatomegaly with a liver span of $15 \mathrm{~cm}$ and signs of free fluid in the abdomen. 


\section{Cureus}
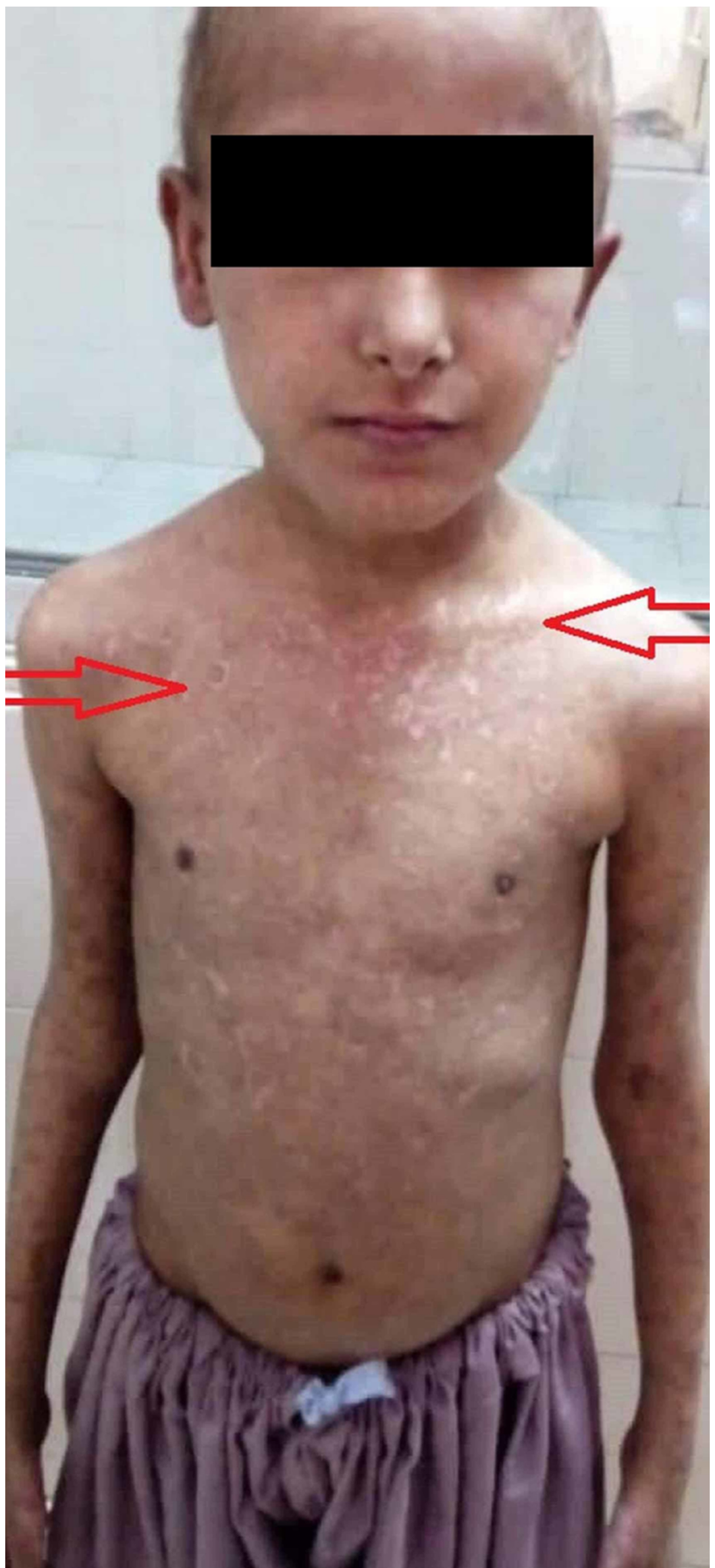

FIGURE 1: Scaly rash with occasional targetoid lesions all over 


\section{Cureus}

\section{the body}

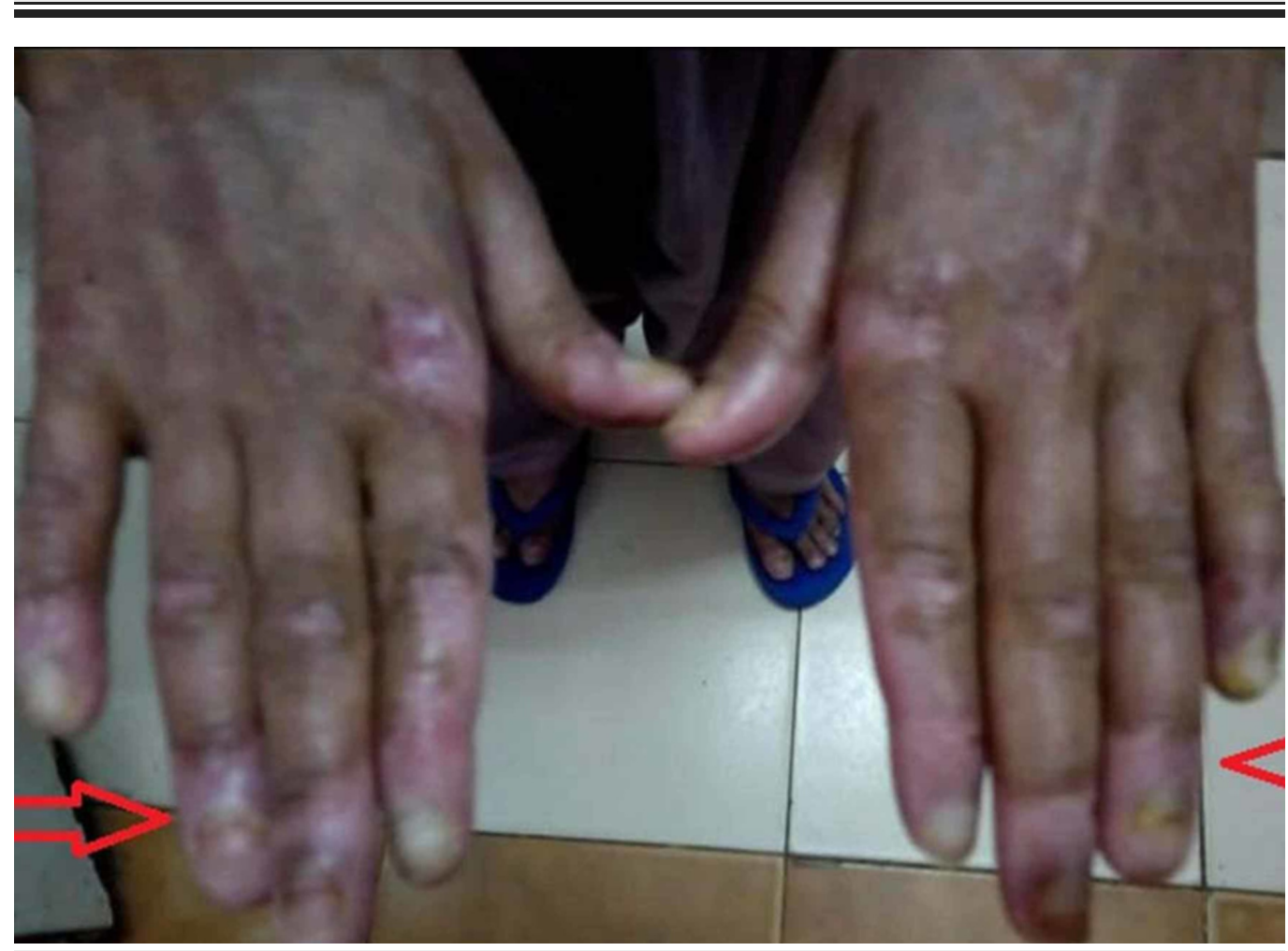

FIGURE 2: Loss of fingernails with peeling of skin

The initial differential diagnosis for the skin rashes and nail dystrophy included SLE, mixed connective tissue disorders, hypohidrotic ectodermal dysplasia, and dyskeratosis congenita. On investigation, a full blood count showed moderate hypochromic, microcytic anaemia with haemoglobin of $8 \mathrm{~g} / \mathrm{dL}$. Raised erythrocyte sedimentation rate (ESR) of $98 \mathrm{~mm} / 1 \mathrm{st} \mathrm{hr}$ and raised C-reactive protein of $7.9(<5 \mathrm{mg} / \mathrm{L})$ were suggestive of inflammation. Serology revealed positive antinuclear antibody (ANA) and positive anti-double-stranded DNA (anti-ds DNA), both suggestive of SLE. Direct Coombs test was also positive. Urine DR showed protein ++, red cell casts and granular casts, which along with an increased ratio of spot urinary protein to creatinine of 4.5, suggesting glomerular involvement. Serum C3 and C4 levels were low, measuring $31 \mathrm{mg} / \mathrm{dL}$ (88-252 mg/dL) and $8 \mathrm{mg} / \mathrm{dL}$ (12-72 mg/dL), respectively, supporting the diagnosis of SLE. Renal and liver function tests were normal except for low serum albumin. Computed tomography (CT) scan of the abdomen confirmed hepatomegaly with marked ascites. X-ray chest showed right-sided LP (Figure 3). CT chest revealed bilateral multifocal multisegmental ground glass haze along with multiple enlarged cervical, pretracheal and carinal lymph nodes, and bilateral mild pleural effusion, extending in the oblique fissure suggestive of active pulmonary infection. 


\section{Cureus}

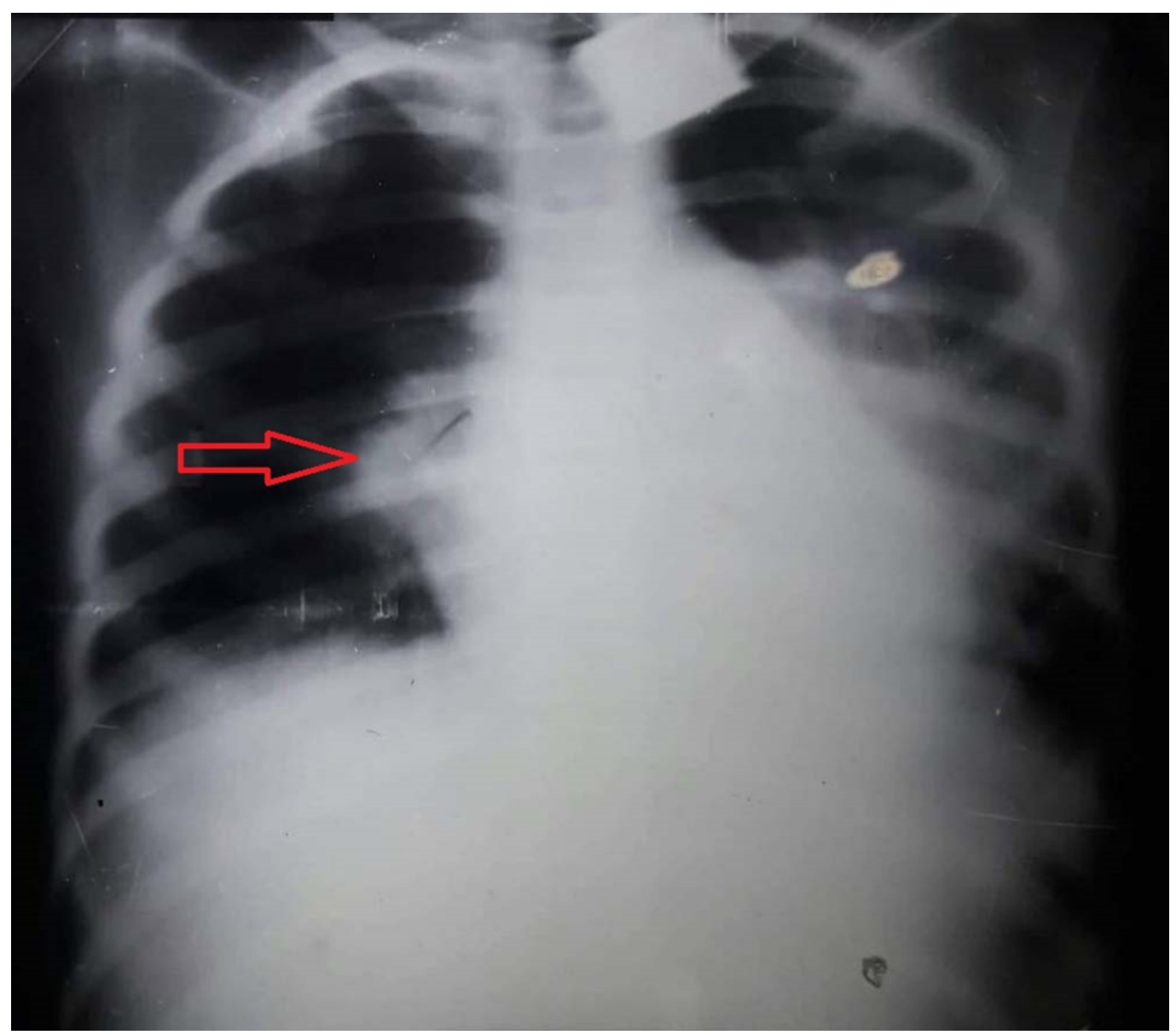

FIGURE 3: X-ray chest showing right-sided lobar pneumonia

Extractable nuclear antigen (ENA) panel revealed a negative anti-Scl-70, negative anti-Jo-1, a negative anti-La (SS-B), positive anti-ribonucleoprotien (RNP), positive anti-Smith (Sm) and a strongly positive anti-Ro (SS-A) antibodies, findings being consistent with the diagnosis of RS. We proceeded with the skin biopsy which revealed interface dermatitis showing lymphocytes and keratinocytes in the epidermis, supporting the diagnosis of erythema multiforme. A diagnosis of RS with lupus nephritis and LP was established based on the clinical manifestations and positive immune-chemistry. The patient was treated with methylprednisolone initially followed by oral prednisolone, azathioprine, naproxen and hydroxychloroquine on the advice of pediatric rheumatologist and antibiotics for pulmonary infection. His rashes improved and proteinuria resolved over a period of two weeks. The patient is under regular follow-up with no recurrence of skin lesions or pneumonia.

\section{Discussion}

Rowell syndrome is a rare disorder characterized by the coexistence of LE with EM. The association was first reported by Scholtz [1]. In 1963, Rowell, Beck and Anderson described 4 female patients who had a similar association. Immunological findings of these patients included a speckled pattern of ANA, positive RF, and the anti-SjT type, which is now considered similar to anti-Ro antibody [2].

Our case meets all the diagnostic criteria of RS, both major and minor making it the first welldefined case of RS from Pakistan. It is important to mention that most of the cases of RS have been reported in middle-aged women; hence the frequency of RS in children is quite rare. A 
similar case was reported in a 15-year-old boy from India; however, our case presented with a unique association of respiratory infection coexistent with the RS which, to our knowledge, has not been reported in the literature so far [5]. These clinical findings, although not a part of RS, could emerge as important associations of the RS in future as more data on RS become available; however, in the present case, this appears to be an incidental finding rather than the consistent feature of the syndrome.

The therapeutic regimen used for RS is similar to that of SLE. Majority of the reported cases showed satisfactory response to corticosteroids with azathioprine or antimalarial drugs, such as chloroquine or hydroxychloroquine [4-6]. Our patient responded well to azathioprine, prednisolone, hydroxychloroquine, and naproxen. A similar case reported another successful treatment option with low-dose cyclosporin A, adding another alternative treatment option for patients refractory to standard therapies [7].

Our literature search also revealed several debates concerning whether RS should be considered as a unique clinical case. Approximately $88 \%$ of the cases report speckled ANA pattern as the most important feature of RS; however, this is shared often with SLE, mixed connective tissue disease and scleroderma. Moreover, the presence of anti-Ro/La antibodies can also be detected in other disorders such as subacute cutaneous lupus erythematosus (SCLE), Sjögren syndrome, SLE, rheumatoid arthritis, and scleroderma [7-9].

\section{Conclusions}

The above-mentioned case adds to the existing scientific literature on RS and highlights the need to consider RS as a differential diagnosis in all patients with LE with EM-like lesions and also the possibility of its occurrence in children in addition to adults. The co-existence of LP in our patient was a novel finding in the setting of RS. Our patient responded well to the conventional treatment strategies employed for RS, indicating the adequacy of the currently accepted therapeutic regimens; however, thorough research needs to be undertaken to establish the existence of RS as a separate entity.

\section{Additional Information \\ Disclosures}

Human subjects: Consent was obtained by all participants in this study. Conflicts of interest: In compliance with the ICMJE uniform disclosure form, all authors declare the following:

Payment/services info: All authors have declared that no financial support was received from any organization for the submitted work. Financial relationships: All authors have declared that they have no financial relationships at present or within the previous three years with any organizations that might have an interest in the submitted work. Other relationships: All authors have declared that there are no other relationships or activities that could appear to have influenced the submitted work.

\section{References}

1. Torchia D, Romanelli P, Kerdel FA: Erythema multiforme and Stevens-Johnson syndrome/toxic epidermal necrolysis associated with lupus erythematosus. J Am Acad Dermatol. 2012, 67:417-421.

2. Rowell NR, Beck JS, Anderson JR: Lupus erythematosus and erythema multiforme-like lesions. A syndrome with characteristic immunological abnormalities. Arch Dermatol. 1963, 88:176180. 10.1001/archderm.1963.01590200064012

3. Lee A, Batra P, Furer V, Cheung W, Wang N: Rowell syndrome (systemic lupus erythematosus + erythema multiforme). Dermatol Online J. 2009, 15:1.

4. Zeitouni NC, Funaro D, Cloutier RA, Gagne E, Claveau J: Redefining Rowell's syndrome. Br J 


\section{Cureus}

Dermatol. 2000, 142:343-346.

5. Bhat RY, Varma C, Bhatt S, Balachandran C: Rowell syndrome. Indian Dermatol Online J. 2014, 5:33-35. 10.4103/2229-5178.144526

6. Tiwary AK, Mishra DK: ANA-negative systemic lupus erythematosus with targetoid lesions: Rowell syndrome or just a rare presentation of lupus erythematosus. J Pak Assoc Dermatol. 2017, 26:269-72.

7. Sharma A, Sharma R: Rowell syndrome - case report and review of literature. Int J Rheumatol Clin Immunol. 2016, 4:CS6. 10.15305/ijrci/v4i1/202

8. Shadid NH, Thissen CA, Van Marion AM, Poblete-Gutiérrez P, Frank J: Lupus erythematosus associated with erythema multiforme: Rowell's syndrome. Int J Dermatol. 2007, 46:30-32. 10.1111/j.1365-4632.2007.03508.x

9. Khandpur S, Das S, Singh MK: Rowell's syndrome revisited: report of two cases from India . Int J Dermatol. 2005, 44:545-549. 10.1111/j.1365-4632.2004.01995.x 